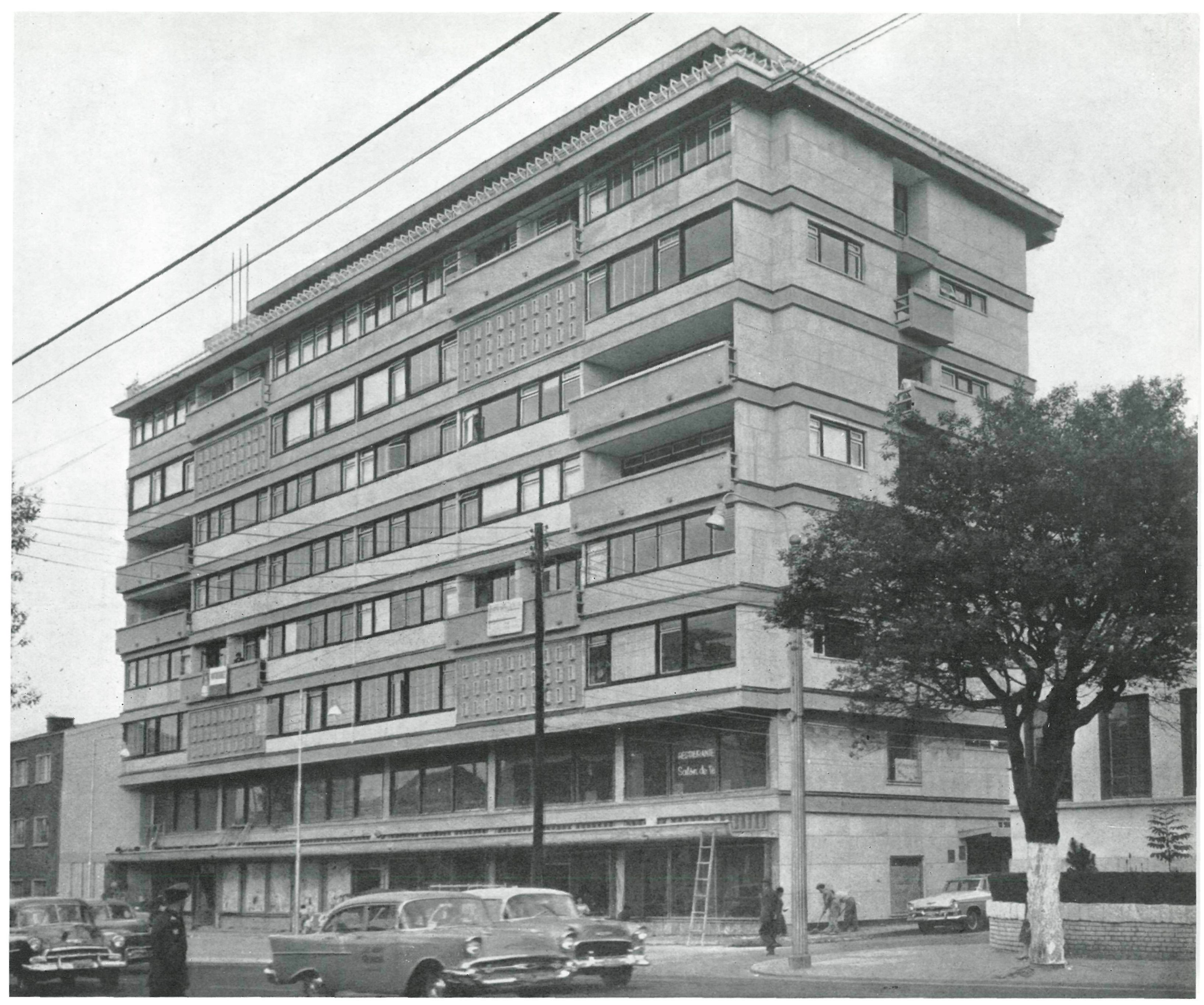

\title{
residencias San Marcos
}

RUBIO, GOMEZ y Cía. Limitada, arquitectos

\section{simopesis}

El inmueble construido está dividido en dos grandes zonas: las plantas bajas destinadas a locales comerciales; y los pisos altos, a apartamentos. Los locales ocupan la casi totalidad de las fachadas, $y$ son de doble o de media altura. Se ha previsto una amplia zona de aparcamiento frente al edificio.

El bloque de apartamentos alterna los simples con los duplex. Este criterio permite disponer una gran variedad de tipos: de un dormitorio, de dos, de tres y de cuatro. Además, se ha conseguido que el ascensor tenga sólo cinco

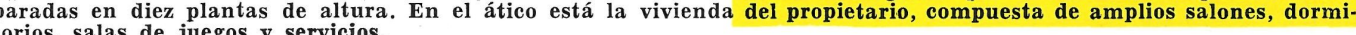

La superficie total de construcción es de $5.100 \mathrm{~m}^{2}$. El edificio tiene 30 apartamentos, además de sus servicios de portería, cuartos de máquinas, incineradores, depósitos de agua, etc. El acabado de las fachadas $s$ ? ha realizado con chapado de piedra, hormigón visto y carpintería metálica, de acero inoxidable.

El número y perfección de los servicios contribuyen a que este conjunto satisfaga adecuadamente el programa previsto y garantice un funcionamiento normal en este tipo de construcciones. 

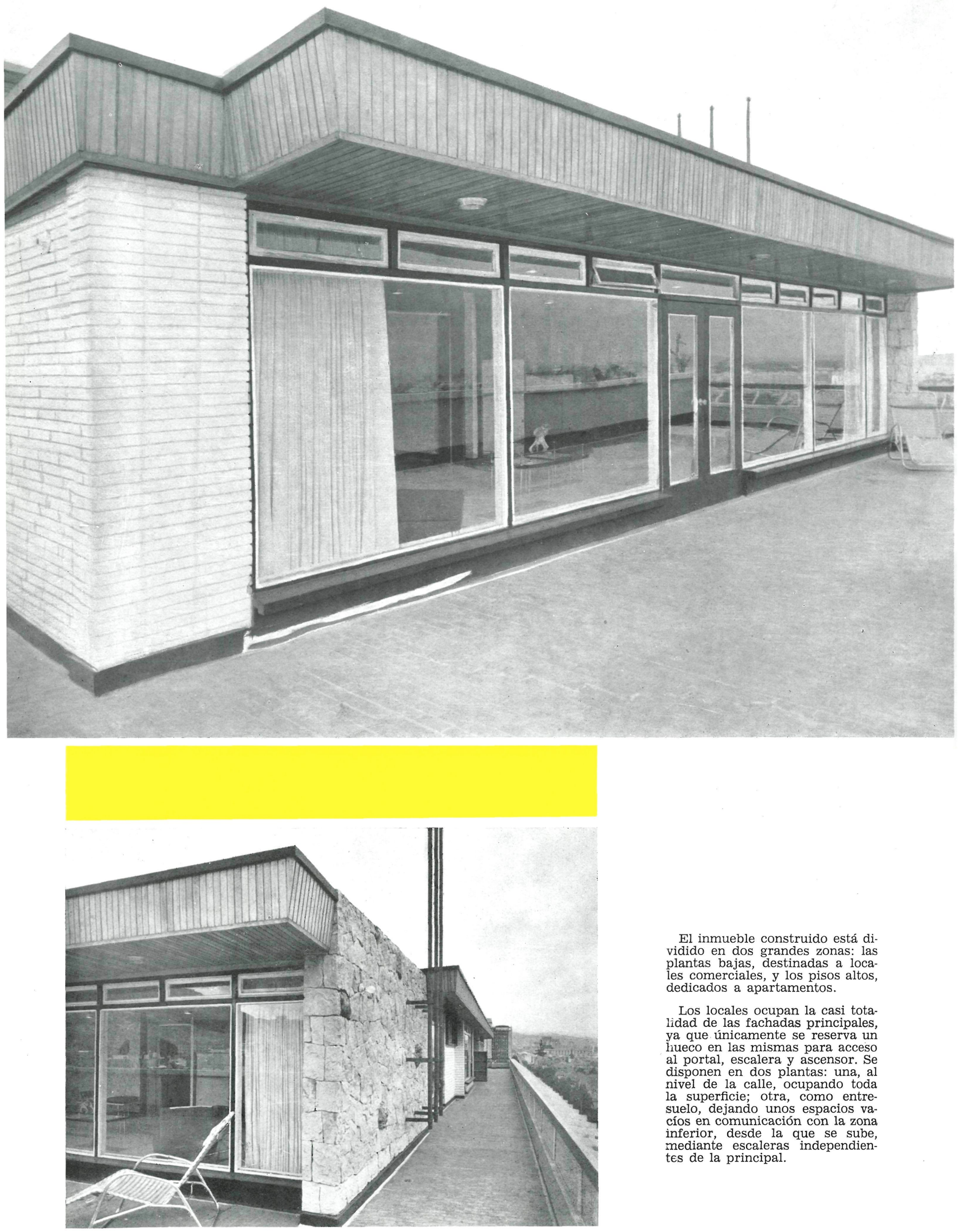

El inmueble construido está dividido en dos grandes zonas: las plantas bajas, destinadas a locales comerciales, y los pisos altos, dedicados a apartamentos.

Los locales ocupan la casi totalidad de las fachadas principales, ya que únicamente se reserva un hueco en las mismas para acceso al portal, escalera y ascensor. Se disponen en dos plantas: una, al nivel de la calle, plantas: una, al la superficie: otra como entrela superficie; otra, como entrecíos en comunicación con la zona cíos en comunicación con la zona inferior, desde la que se sube, mediante escaleras 
p
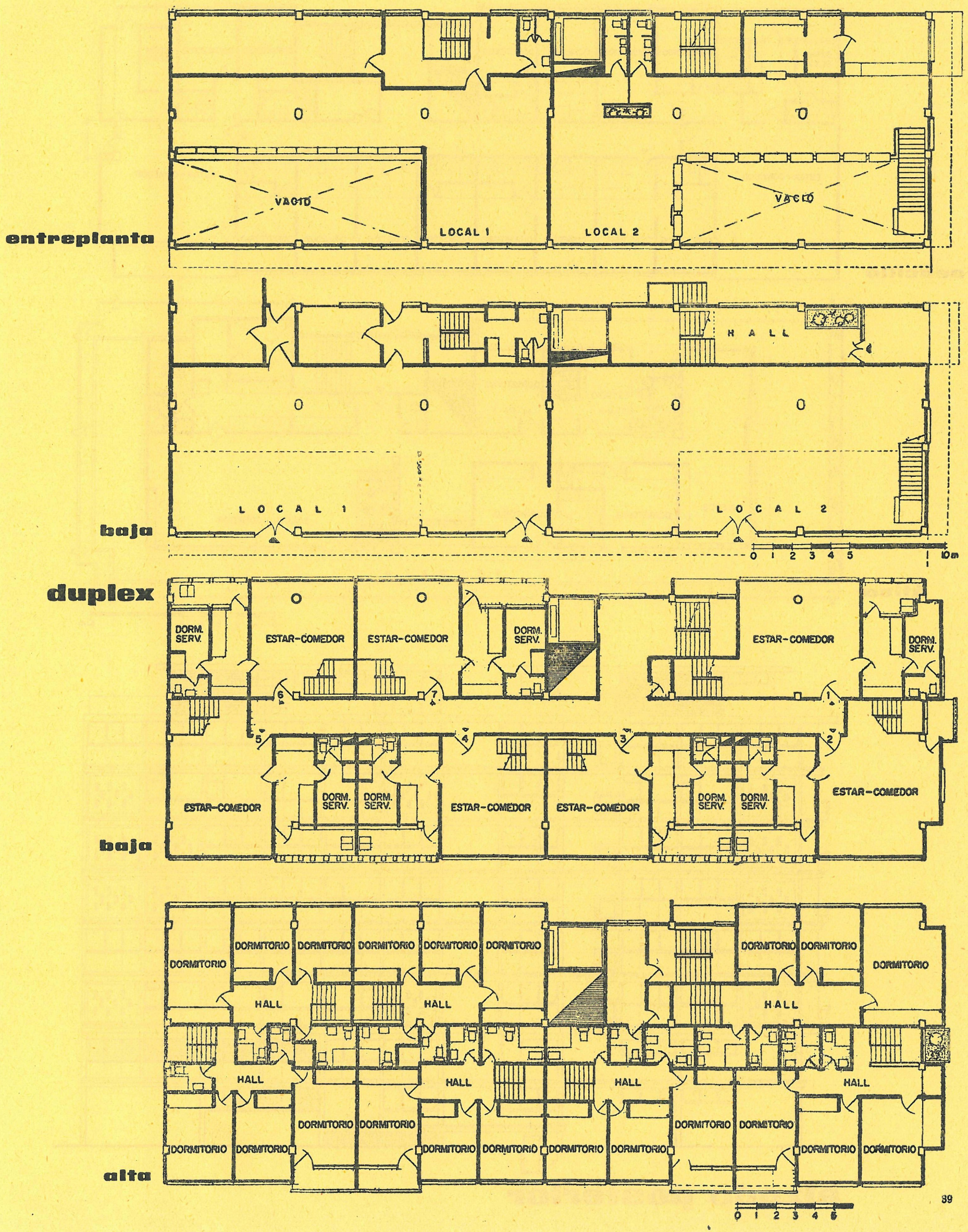


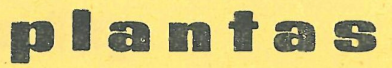
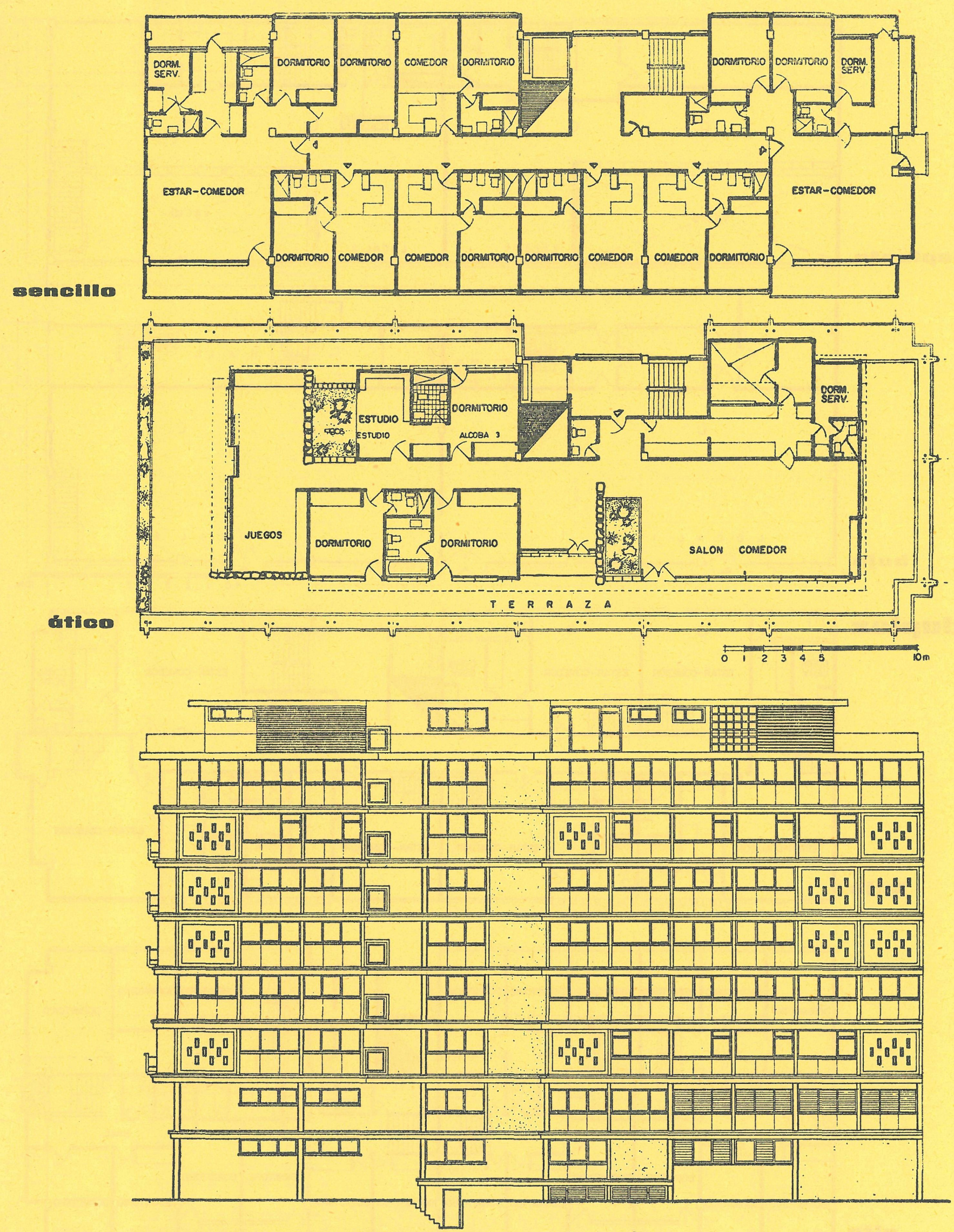

alzado posterior 

edificio, una amplia zona de estacionamiento de vehículos.

El bloque de apartamentos alterna los simples con tos alterna los simples con permite disponer una gran vermite disponer una gran dormitorio, de dos, de tres y de cuatro.

En líneas generales, el inmueble, además de la zona dedicada a locales comerciales, consta de seis plantas de pisos, $\mathrm{y}$ ático.

De estas plantas, las dos primeras y las dos últimas, con duplex, y las dos in termedias, tercera y cuarta, con apartamentos simta,

Dentro de los simples, apreciamos las siguientes variantes: una constituida por vestíbulo, sala estar comedor reducida, dormitorio, baño y cocina; otra con vestíbulo, sala estarcomedor de amplias dimensiones, tres dormitorios, cocina, baño y aseo.

Los duplex agrupan en la planta inferior la sala de estar amplia, cocina con lavadero, dormitorio y lavadero, de servicio; mientras aseo de servicio; mientras que en la parte superior, a la que se accede desde la dormitorios y bano.
Se ha previsto, frente al

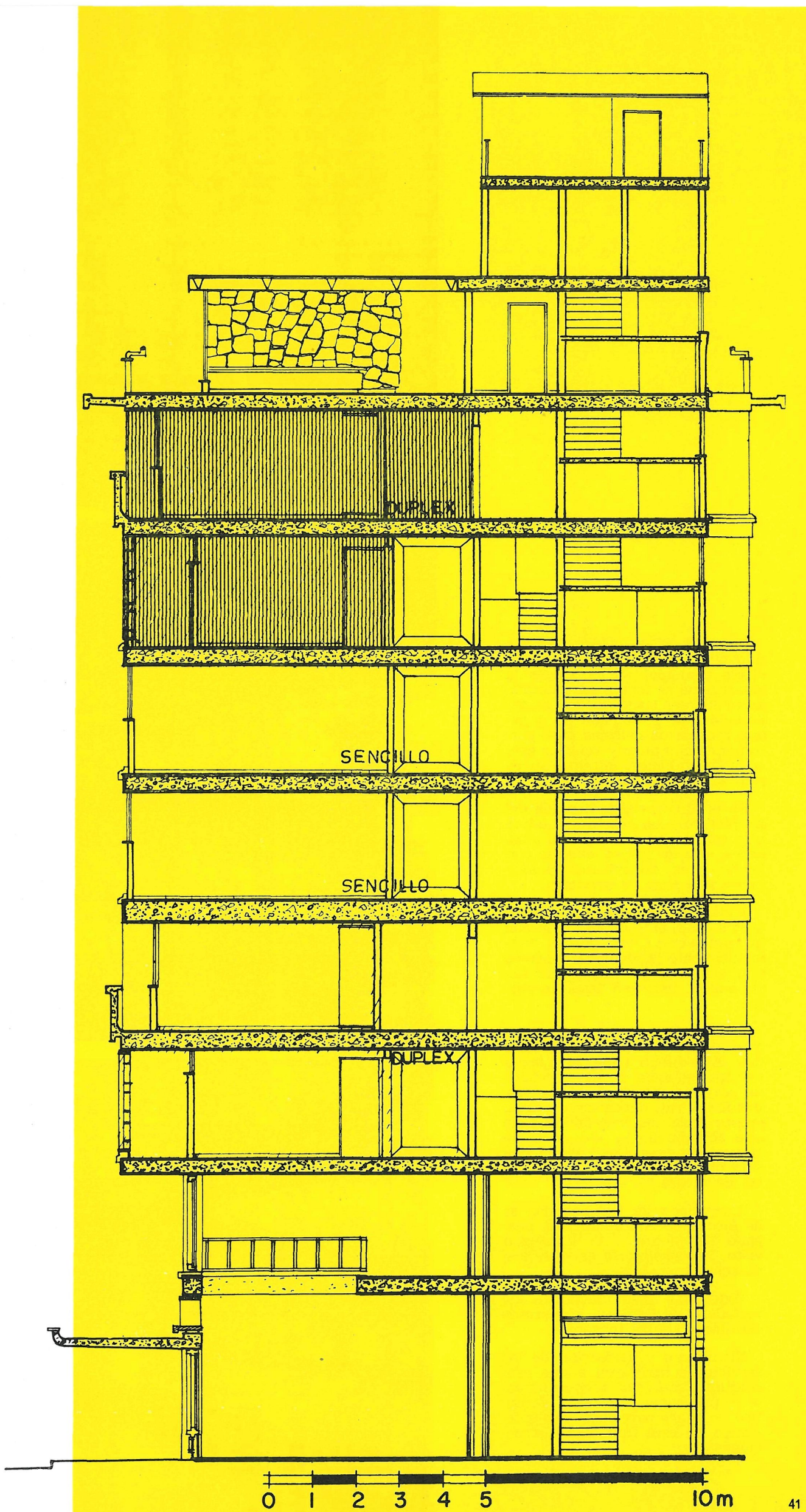




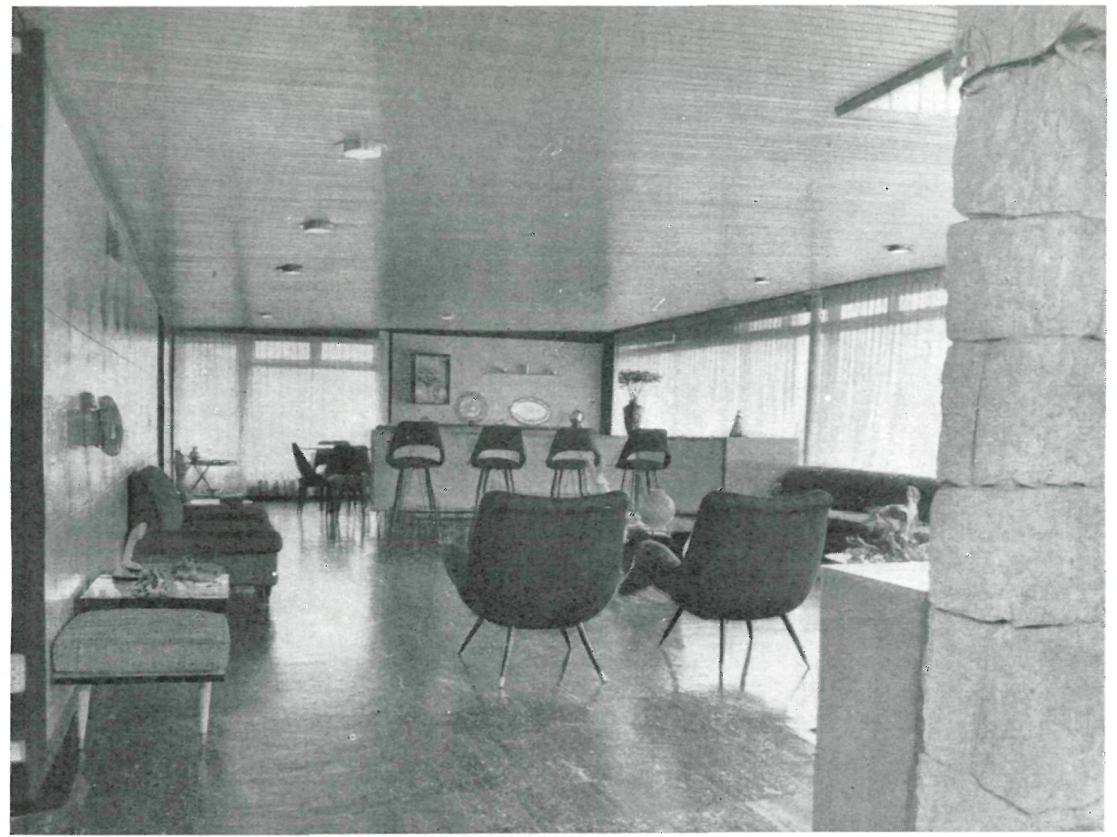

En la zona de cocinas de los duplex, perteneciente a la fachada principal, se aprovecha la galería que se forma para emplazamiento del lavadero. En la decoración de la fachada se dejan una especie de celosías que, per. mitiendo iluminar y ventilar esta zona de servicios, impidan su visión desde el exterior.

En el ático está la vivienda del propietario, compuesta de un ampropietario, compuesta de u plio salón-comedor, salones auxiliares, sala de juegos, estudio con pales y la zona de servicios.

Todas las habitaciones importantes están en comunicación con la gran terraza, que bordea perimetralmente la vivienda, y ofrece el aliciente de las magníficas vistas que se disfrutan desde la misma.

La superficie total de construcción es de 5.100 metros cuadrados. El edificio tiene 30 apartados. El edificio tiene 30 aparta-
mentos, además de sus servicios mentos, además de sus servicios incineradores, depósitos de agua, cuarto para las instalaciones de aire acondicionado, etc., y se ha conseguido que el ascensor tenga sólo cinco paradas en diez plantas de altura.

El acabado de las fachadas se ha ejecutado combinando el chapado de piedra y el hormigón visto. La carpintería es metálica, de acero inoxidable.

Todos los pisos disponen de vertedero de basuras, y aparatos extintores contra incendios.

El número y perfección de los servicios contribuyen a que este conjunto satisfaga adecuadamente el programa previsto y garantice un funcionamiento excelente, dentro de este tipo de construcciones.
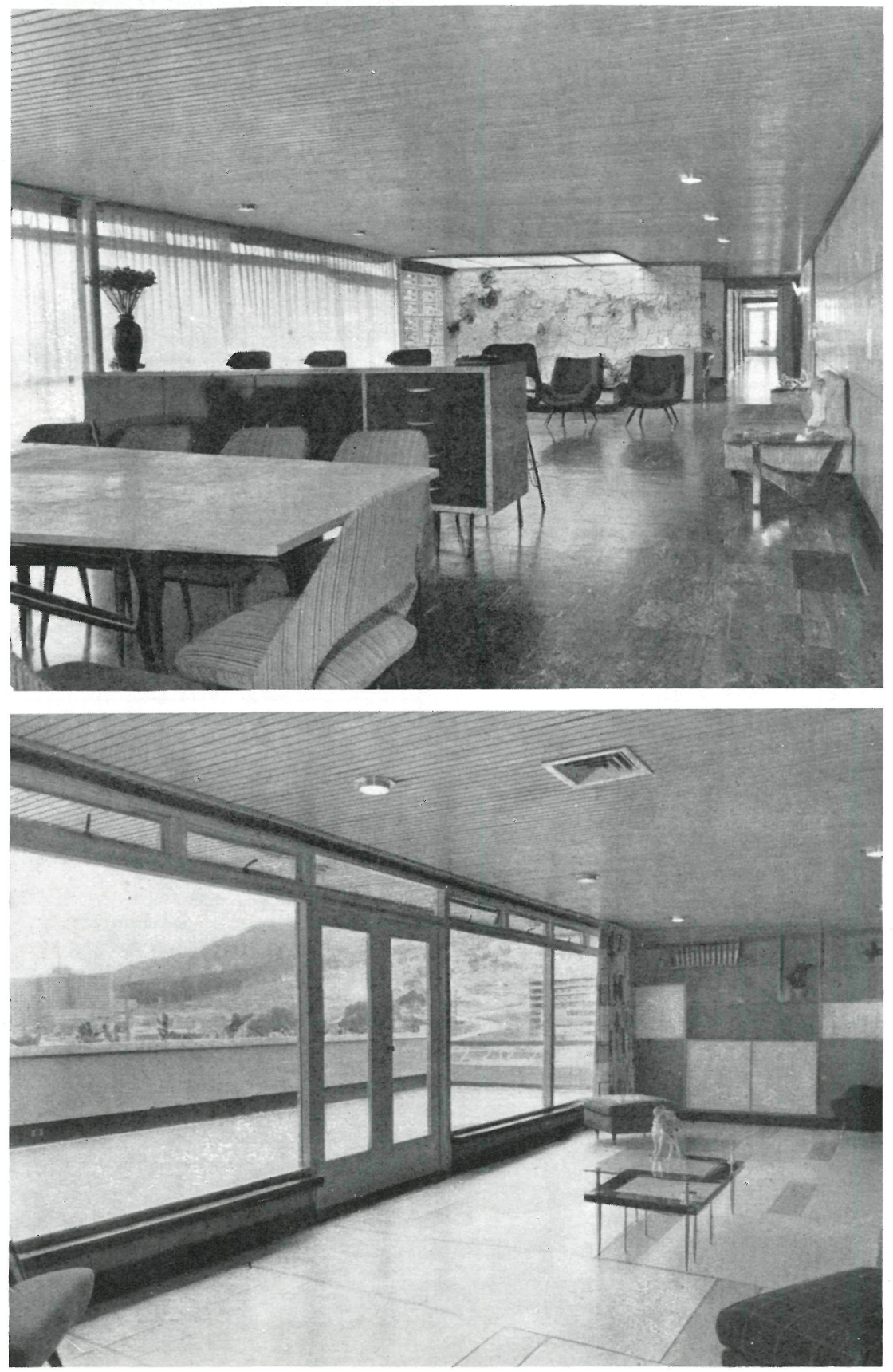


\section{Rébidemces San Marcos}

Rubio, Gómez \& Cie. Limitada, architectes.

L'immeuble construit est divisé en deux grandes zones: les rez-de-chaussée destinés à des locaux commerciaux et les étages supérieurs à des appartements. Les locaux occupent presque toutes les façades et sont de hauteur double ou moyenne. Une ample zone de stationnement a été prévue en face de l'édifice.

Le bloc d'appartements alterne les simples et les Duplex. Ce système permet de disposer une grande variété de types: d'une, deux, trois ou quatre chambres. L'ascenseur n'a que 5 arrêts pour 10 étages. Le dernier de ces étages a été réservé au propriétaire et se compose d'amples salons, chambres, salles de jeux et services.

La surface totale de construction est de 5.100 mètres carrés. L'édifice comprend 30 appartements, en plus des locaux réservés au concierge, salles de machines, incinérateur, dépôts, d'eau, etc. Les façades ont été réalisées à l'aide de placages de pierre, béton vu et menuiserie métallique en acier inoxidable. Le nombre et la perfection de ses services font que cet ensemble réalise le programme prévu et garantisse un fonctionnement normal pour ce type de constructions.

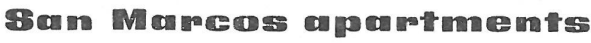

Rubio, Gomez \& Gia., Ltd., architects.

This house is divided into two main zones. One of them is the ground floor, which is devoted to business purposes. The other consists of the remaining floors, which contain apartments. An ample parking area has been provided in front of the building.

The flats are, alternatively, simple, and so called duplex apartments. This basic design allows for a great variety of particular arrangements, including one, two, three or four bedrooms. It has been arranged for the lift to have only five stops for the ten floor levels. The owner lives in the attic, which contains large living rooms, bedrooms, amusement facilities and services.

The total built up area is 5,100 $\mathrm{ms}^{2}$. The building contains 30 apartments, as well as porter's quarters, an engine room, incinerators, water tanks, etc. The outer walls are faced in stone, and concrete, and doors and windows are of stainless steel.

The type and quality of the services ensure that this building provides every reasonable comfort to its occupants.

\section{Molnmumgen Ben Marco}

Rubio, Gómez \& Cía. Limitada, Architekten.

Der gebaute Besitz ist in zwei grosse Teile geteilt: die unteren Geschosse sind für Geschäftsräume bestimmt und die oberen für Wohnungen. Die Räume nehmen fast die ganze Fassadenseite ein und sind von doppelter oder halber Höhe. Gegenüber dem Gebäude wurde eine weite Zone als Parkplatz vorgesehen.

Der Block der Wohnungen wechselt die einfachen mit den doppelten. Dieser Gesichtspunkt erlaubt, über eine grosse Verschiedenheit von Typen zu verfügen: mit einem Schlafraum, mit zweien, drei oder vier. Ausserdem hat man erreicht, dass der Aufzug nur 5 Stillstände in 10 Stockwerken hat. Ganz oben hat der Besitzer seine Wohnung, die aus grossen Sälen, Schlafräumen, Spielzimmern und Badezimmern besteht.

Die Gesamtoberfläche des Baues beträgt $5.100 \mathrm{~m}^{2}$. Das Gebäude hat 30 Wohnungen, abgesehen von den Pförtnereien, Maschinenräumen, Heizungsanlagen, Wasserbehältern, u.s.w. Die Fertigung der Fassaden wurde mit Steinplatten durchgeführt, weiters mit Sichtbeton und Metalltischlerei aus rostfreiem Stahl. Die Anzahl und Vollkommenheit der Betriebe tragen dazu bei, dass dieses Ganze das vorgesehene Programm entsprechend zufriedenstellt und einen normalen Gang in dieser Bauart garantiert. 